\title{
Treatment of Textile Effluent Using Sacrificial Electrode
}

\author{
K.Chithra \\ R.Thilakavathi \\ A. Arul Murugan \\ C. Marimuthu \\ N.Balasubramanian (corresponding author) \\ Department of Chemical Engineering, A.C. Tech Campus \\ Anna University-Chennai, Chennai-600 025, India \\ Tel: 91-44-2220-3501Ｅ-mail: nbsbala@annauniv.edu
}

\begin{abstract}
Experiments were carried out to treat synthetic Bismarck Brown dye effluent using electro coagulation covering a wide range in operating conditions. The influence of operating parameters on efficiency of the process has been critically examined. The batch experimental results showed that the dye effluent can be effectively treated using electro coagulation. The overall COD removal efficiencies has been obtained $99 \%$, under optimum operating condition. Further it is attempted to fit the experimental data with popular adsorption isotherms Langmuir and Temkin models. The predictions of Langmuir adsorption isotherm model are in good agreement with the experimental data.
\end{abstract}

Keywords: Electro coagulation, Wastewater treatment, COD removal, Sacrificial electrode

\section{Introduction}

Effluents with sturdy color and high COD are common in chemical process industries such as textile, paper, leather and mineral processing industries. In textile industries, dyeing and finishing are the two most important process operations which consumes large amount of water and generates considerable amount of wastewater which contains strong color, suspended particles, high $\mathrm{pH}$ and high COD and BOD. The textile effluent cannot be treated by chemical methods as these techniques generate considerable amount of sludge, which itself requires further treatment. While the biological methods are cheap and simple to apply, but cannot be applied to textile wastewaters since most of the commercial dyes are non-biodegradable and toxic to the organisms resulting in sludge bulking. On the other hand the advanced techniques such as reverse osmosis/ultra-filtration have the disadvantages of high cost and low throughput.

In recent years, there has been increasing interest in the use of electrochemical techniques such as electro coagulation, electro flotation, electro decantation, electrochemical oxidation, catalytic wet air oxidation etc for the treatment of dye house effluent. Among these techniques, electro coagulation emerges as one of the promising techniques for the treatment of organic effluent treatment. Successful electrocoagulation (EC) treatment of various industrial effluents has been reported by several researchers as it is considered to be potentially an effective tool for treatment of wastewaters with high removal efficiency. Electro coagulation has been adopted successfully to treat various industrial effluents such as potable water (Viek et al., 1984); oil mill wastewater (Inan et al., 2004); urban wastewater ( Pouet and Grasmick, 1995); heavy metal laden wastewater (Lai and Lin, 2003); nitrite effluent (Koparal and Ogutveren, 2002); defluoridation (Zhu et al., 2007); arsenic removal (Balasubramanian and Madhavan, 2001); textile industries (Can et al., 2003); landfill leachate (Tsai et al., 1997); restaurant wastewater (Chen et al., 2000); salina wastewater (Lin et al., 1998); tar sand and oil shale wastewater (Renk, 1988); laundry wastewater (Ge et al., 2004).

The objective of the present study is to investigate the treatment of Bismarck Brown dye house effluent through electro coagulation under various operating conditions. The mechanism of electro coagulation and the effect of individual parameters on the efficiency of electro coagulation have been critically examined.

\section{Mechanism of Electrocoagulation}

It is known fact that electro coagulation is a complex and interdependent process. The sacrificial anode produces coagulating agent to dose the wastewater while electrolytic gases are generated at the cathode. The process of electro coagulation involves three successive steps:

i. Formation of coagulants by electrolytic oxidation of the 'sacrificial electrode' 


\section{ii. Destabilization of the contaminants, particulate suspension, and breaking of emulsions and \\ iii. Aggregation of the destabilized phases to form flocs.}

The dissolved $\mathrm{Fe} / \mathrm{Al}$ in the electrolyte generates corresponding metal ions and hydrolyzes to polymeric iron or aluminum hydroxide, which are excellent coagulating agents. The sacrificial anode continuously produces polymeric hydroxides at the anode and coagulation occurs when these metal cations combine with the negative charge particles carried toward the anode by electro phoretic motion. In the present case aluminum has been used as anode and the electrode reaction can be written as (Chen, 2004)

Anode: $A l(s) \rightarrow A l^{3+}(a q)+3 e^{-}$

Cathode: $3 \mathrm{H}_{2} \mathrm{O}(\mathrm{l})+3 e^{-} \rightarrow \frac{3}{2} \mathrm{H}_{2}(g)+3 \mathrm{OH}^{-}(a q)$

$\mathrm{Al}^{3+}$ and $\mathrm{OH}^{-}$ions generated during the electrochemical reactions react to form various monomeric and polymeric species and transform into $\mathrm{Al}(\mathrm{OH})_{3}$ according to the following reaction.

$A l^{3+}(a q)+3 \mathrm{H}_{2} \mathrm{O}(l) \rightarrow \mathrm{Al}(\mathrm{OH})_{3}(s)+3 \mathrm{H}^{+}(a q)$

These $\mathrm{Al}(\mathrm{OH})_{3}$ flocs capture the dye molecules present in the solution by the following reaction mechanism

Precipitation:

$\mathrm{DYE}+\mathrm{Al}_{\mathrm{m}} \rightarrow\left[\mathrm{DYE} \mathrm{Al} \mathrm{m}_{\mathrm{s}}\right.$

$\mathrm{DYE}+\mathrm{Al}_{\mathrm{p}} \rightarrow\left[\mathrm{DYE} \mathrm{Al} \mathrm{p}_{\mathrm{s}}\right.$

Adsorption:

$\mathrm{DYE}+\mathrm{Al}(\mathrm{OH})_{3},{ }_{\mathrm{s}} \rightarrow \mathrm{Al}(\mathrm{OH})_{3, \mathrm{~s}}(D Y E)$

(6)

$\left[\mathrm{DYE} \mathrm{Al}_{\mathrm{p}}\right]_{\mathrm{s}}+\mathrm{Al}(\mathrm{OH})_{3, \mathrm{~s}} \rightarrow\left\lfloor\mathrm{Al}(\mathrm{OH})_{3} \mathrm{DYEAl}_{\mathrm{p}}\right\rfloor_{\mathrm{s}}$

In the electro coagulation process, the rate of removal of COD is proportional to the pollutant concentration and the amount of aluminium hydroxide formed.

$\frac{-d[\mathrm{COD}]}{d t}=k[\mathrm{COD}]\left[\mathrm{Al}(\mathrm{OH})_{3}\right]$

Since the generation of aluminium hydroxide produced can be assumed constant for a given current density, the equation (8) can be simplified to pseudo first order kinetics as

$\frac{-d[C O D]}{d t}=k[C O D]$

The integration of equation (9) results

$\log \frac{[C O D]_{t}}{[C O D]_{o}}=-k t$

The slope of the plot $\log [\mathrm{COD}]_{\mathrm{t}} /[\mathrm{COD}]_{0}$ versus time gives rate constant.

\section{Experimental}

Experiments were carried out in a batch electrochemical reactor of $250 \mathrm{ml}$. Aluminium and Stainless Steel of $7 \times 4 \mathrm{~cm}^{2}$ were used as anode and cathode respectively. The electrodes were washed with dilute acid to remove surface grease/other impurities on the metal surface. The experiments were carried out under potentiostatic conditions covering wide range in operating conditions and samples were collected periodically for analyzed. The anode was weighed before and after the experiment for each experiment run to estimate the electrode consumption. At the end of the each run the solution was centrifuged at $2500 \mathrm{rpm}$, filtered and the filtrate was analyzed. The pollutant degradations were estimated by Colorimeter by standard estimation procedure for COD estimation.

\section{Adsorption Isotherm}

As stated earlier, the generated $\mathrm{Al}(\mathrm{OH})_{3}$ flocs remains as a suspension in the electrolyte solution which effectively remove the pollutants by adsorption resulting in charge neutralization by complexation/ electrostatic attraction. It is attempted to extend the adsorption isotherms $\mathrm{Al}(\mathrm{OH})_{3}$ for $\mathrm{COD}$ removal. The electrode consumption can be estimated according to Faraday's Law while the amount of flocs can be estimated stoichiometrically. The most widely used adsorption isotherms Langmuir and Temkin isotherms have been attempted in the present investigation to check the adsorption kinetics.

The mathematical expression of Langmuir isotherm can be written as 
$\frac{C_{e}}{Q_{e}}=\frac{1}{K_{L}}+\frac{a_{L}}{K_{L}} C_{e}$

where the constant $K_{\mathrm{L}}$ refers Langmuir equilibrium constant. A plot of $C_{\mathrm{e}} / Q_{\mathrm{e}}$ versus $C_{\mathrm{e}}$ gives a straight line results $a_{\mathrm{L}} / K_{\mathrm{L}}$ (slope) and $1 / K_{\mathrm{L}}$ (intercept).

The Temkin isotherm can be expressed mathematically as

$Q_{e}=\frac{R T}{b} \ln A+\frac{R T}{b} \ln C_{e}$

$\frac{R T}{b}=B$

where $\mathrm{A}$ and $\mathrm{B}$ are Temkin constants. A plot of $Q_{\mathrm{e}}$ versus $\ln C_{\mathrm{e}}$ enables to determine the constants $A$ and $B$.

\section{Results and Discussion}

Experiments were carried out at various $\mathrm{pH}$ by adjusting the electrolyte with sodium hydroxide or hydrochloric acid and the observations are given in Figures 1-5. It can be ascertained from the Figure 1 the percentage COD removal increases with increase in the process time. The rate of increase in the percentage COD removal is high in the beginning of the process and approaches monotonical situation beyond 30mintutes of process time. This can be explained that the more of coagulation process occurs within 30minutes of process time. This can be explained that the existence of excess colloids for the adsorption at high initial concentrations.

The Figure 2 shows the effect of electrolyte $\mathrm{pH}$ on percentage COD removal efficiency. It can be seen from the figure that the percentage COD removal increases with an increase in the electrolyte $\mathrm{pH}$ from 3 to 4 . However no significant improvement has been observed when the $\mathrm{pH}$ increased beyond $\mathrm{pH}$ value of 4 . A maximum $97 \%$ removal efficiency has been observed at a $\mathrm{pH}$ value of 4 in the present investigation.

The effect of applied charge on COD removal efficiency is given in Figure 3. It can be seen from the figure that the percentage COD removal efficiency increases with an increase in the applied current density. This can be explained that at high current densities, the extent of anodic dissolution of aluminum increases, resulting in a greater amount of precipitate for the removal of pollutants. In addition, the rate of bubble generation also increases with increasing current density, which enhances the COD removal efficiency.

The reaction rate constant, $k$, was estimated from a plot of $\ln \left[\mathrm{COD}_{t} / \mathrm{COD}_{0}\right]$ versus electrolysis time. The influence of operating parameters on the reaction rate constant has been verified and it has been observed that the rate constant, $\mathrm{k}$, is influenced by the applied charge [Figure 4.]. However no significant improvement has been observed with electrolyte $\mathrm{pH}$.

Figure 5 shows the relationship between the amount of COD adsorbed per unit mass of charged hydroxo cationic complexes $\left(Q_{\mathrm{e}}\right)$ and final COD in the aqueous phase $\left(C_{\mathrm{e}}\right)$. The plots of COD uptake against equilibrium concentration indicate that adsorption increases with concentration and approaches saturation. Based on the correlation coefficient $\left(\mathrm{r}^{2}\right)$ shown in Table 1, the adsorption isotherm with hydroxo cationic complexes can be described by the Langmiur equation (11) which gives a better fit of experimental data than the Temkin equation (12).

\section{Conclusions}

Experiments were carried out to treat dye house effluent using electro coagulation. The influence of initial dye concentration, $\mathrm{pH}$ and current density on coagulation efficiency has been critically examined. It has been observed from the present investigation that the percentage of COD reduction is significantly influenced by the initial dye concentration, $\mathrm{pH}$ and current density. The adsorption isotherms namely Langmuir and Temkin isotherms were used to check the adsorption kinetics.

\section{References}

Balasubramanian, N., Madhavan., K. (2001). Arsenic removal from industrial effluent through electrocoagulation. Chemical Engineering Technology 24, 519-521.

Can, O.T., Bayramoglu, M., Kobya, M. (2003). Decolorization of reactive dye solutions by electrocoagulation using aluminum electrodes, Ind. Eng. Chem. Res. 42, 3391-3396.

Chen, G. (2004). Electrochemical technologies in wastewater treatment, Sep. Purifi. Technol., 38, 11-41.

Chen, X., Chen, G., Yue, P.L. Separation of pollutants from restaurant wastewater by electrocoagulation, Sep. Purif. Technol. 19 (2000), pp. 65-76.

Ge, J., Qu, J., Lei, P., Liu, H., 2004. New bipolar electro coagulation- electro flotation process for the treatment of laundry wastewater. Sep. Purif. Technol. 36, 33-39. 
Inan, H., Dimoglo, A., Şimşek, H., Karpuzcu, M. (2004). Olive oil mill wastewater treatment by means of electro-coagulation, Separat. Purif. Technol. 36, 23-31.

Koparal, A..S., Ogutveren, U.B. (2002). Removal of nitrate from water by electroreduction and electrocoagulation, $J$. Hazard. Mater. 89, 83-94.

Lai., C.L., Lin, S.H. (2003). Electrocoagulation of chemical mechanical polishing (CMP) wastewater from semiconductor fabrication, Chem. Eng. J. 95, 205-211.

Lin, S.H., Shyu, C.T., Sun, M.C., (1998). Saline wastewater treatment by electrochemical method. Water res. 32, 1059-1066.

Pouet, M.F., Grasmick, A. (1995). Urban wastewater treatment by electrocoagulation and flotation, Water Sci. Technol. 31, 275-283.

Renk, R.R., 1988. Electrocoagulation of tar sand and oil shale wastewater. Energy Prog. 8, 205-208.

Tsai, C.T., Lin, S.T., Shue, Y.C., Su, P.L., 1997. Electrolysis of soluble organic matter in leachate from landfills. Water Res. 31, 3073-3081.

Vik., E, Carlson., D, Eikum., A. (1984). Electrocoagu-lation of potable water. Water Research, 11, 1355-1600.

Zhu, J., Zhao, H., Ni, J. (2007). Fluoride distribution in electrocoagulation defluoridation process Separation and Purification Technology, 56, 2, 184-191.

Table 1. Isotherm constants for adsorption of $\mathrm{COD}$ on $\mathrm{Al}(\mathrm{OH})_{3}$

\begin{tabular}{|l|c|c|c|}
\hline Langmuir & $\mathrm{K}_{\mathrm{L}}\left(\mathrm{Lg}^{-1}\right)$ & $\mathrm{a}_{\mathrm{L}}\left(\mathrm{Lmg}^{-1}\right)$ & $\mathrm{r}^{2}$ \\
\hline & $8.1 \times 10^{-6}$ & 0.0059 & 0.9613 \\
\hline Temkin & $\mathrm{B}$ & $\mathrm{A}\left(\mathrm{Lg}^{-1}\right)$ & $\mathrm{r}^{2}$ \\
\hline & $3 \times 10^{5}$ & 0.006 & 0.92 \\
\hline
\end{tabular}

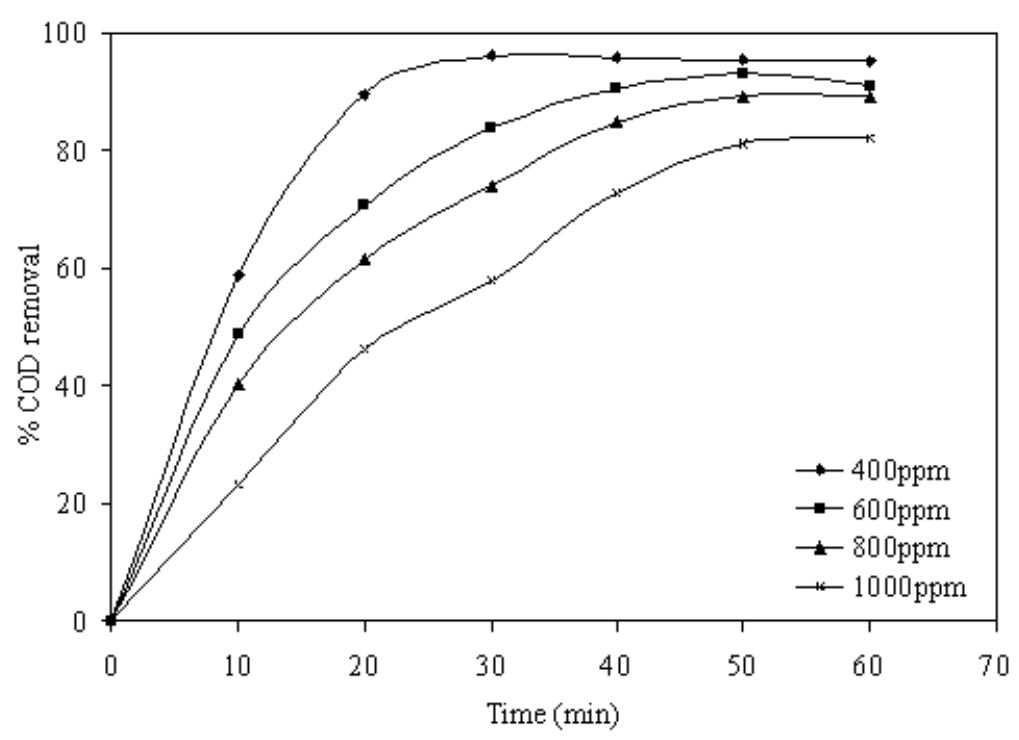

Figure 1. Effect of electrolysis time on percentage COD removal

(Current density: $6 \mathrm{mAcm}^{-2}$; Supporting electrolyte concentration: 400ppm). 


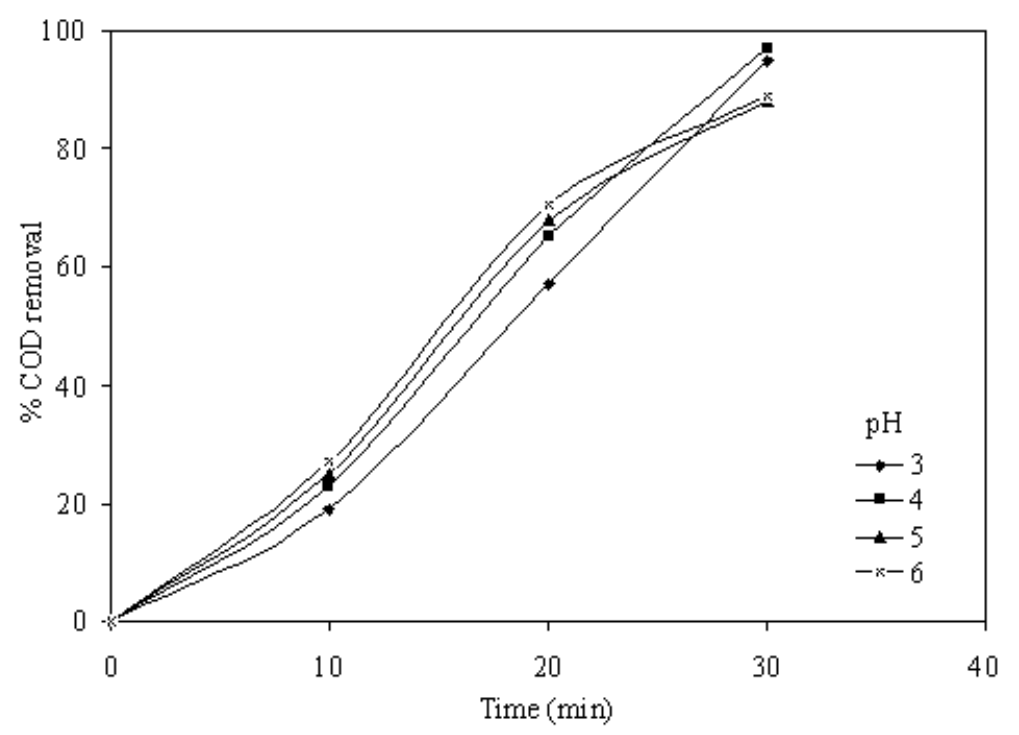

Figure 2. Effect of initial $\mathrm{pH}$ on percentage COD removal

(Supporting electrolyte concentration: 400ppm; Current density: $6 \mathrm{mAcm}^{-2}$; Electrolysis time: $1 \mathrm{hr}$ ).

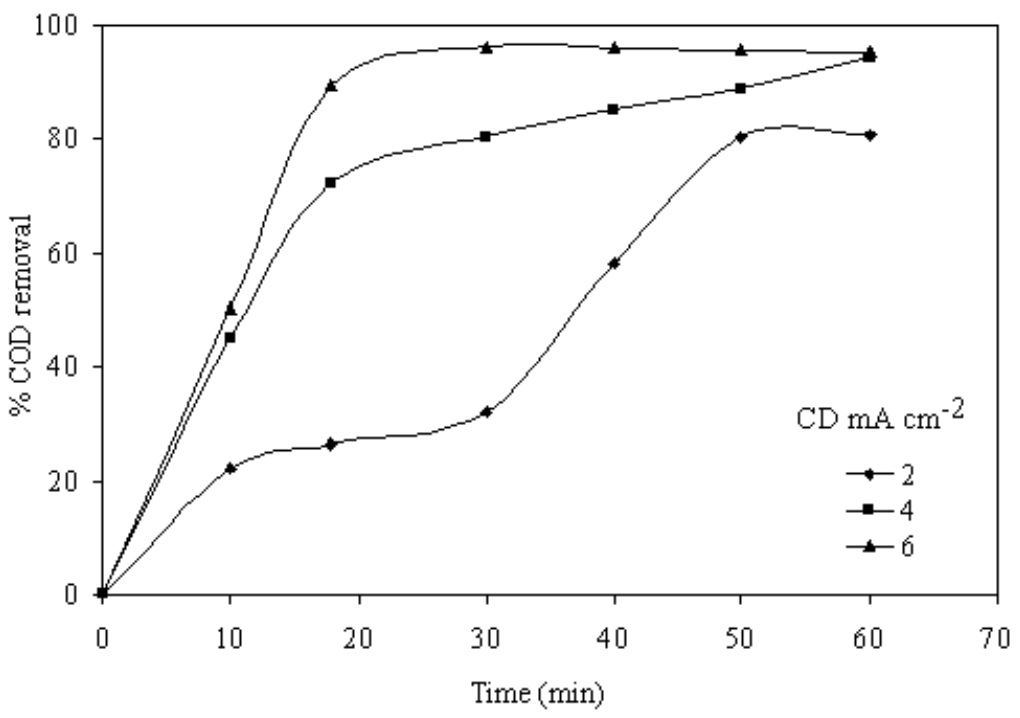

Figure 3. Effect of current density on percentage COD removal

(Electrolysis time: 30min; Supporting electrolyte concentration: 400ppm). 


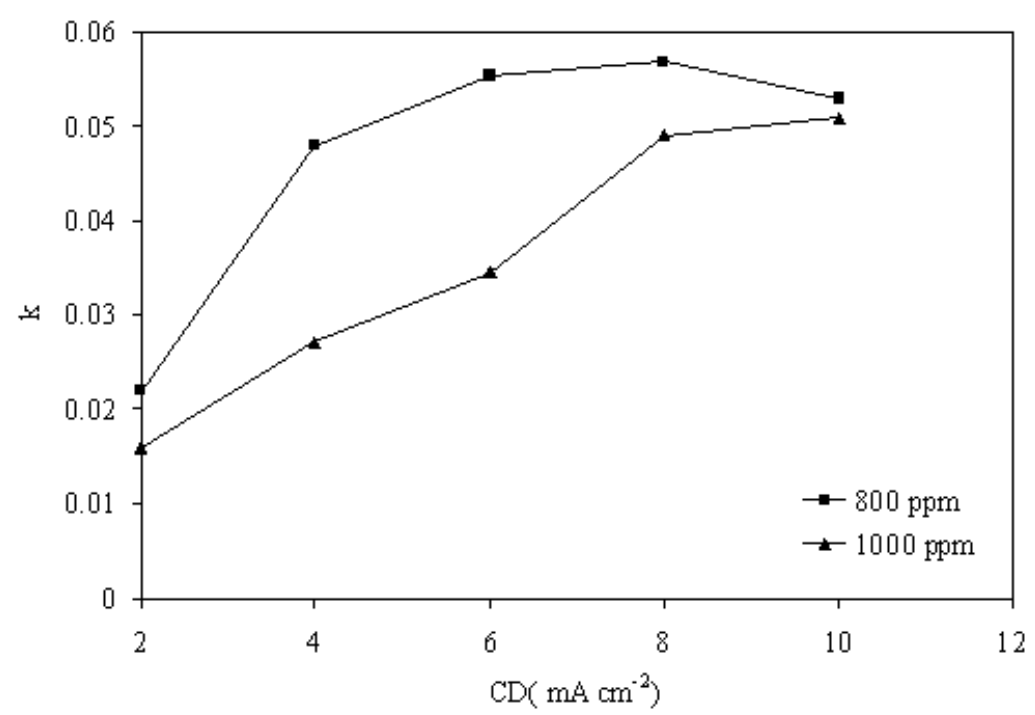

Figure 4. Effect of current density on rate constant $\mathrm{k}$.

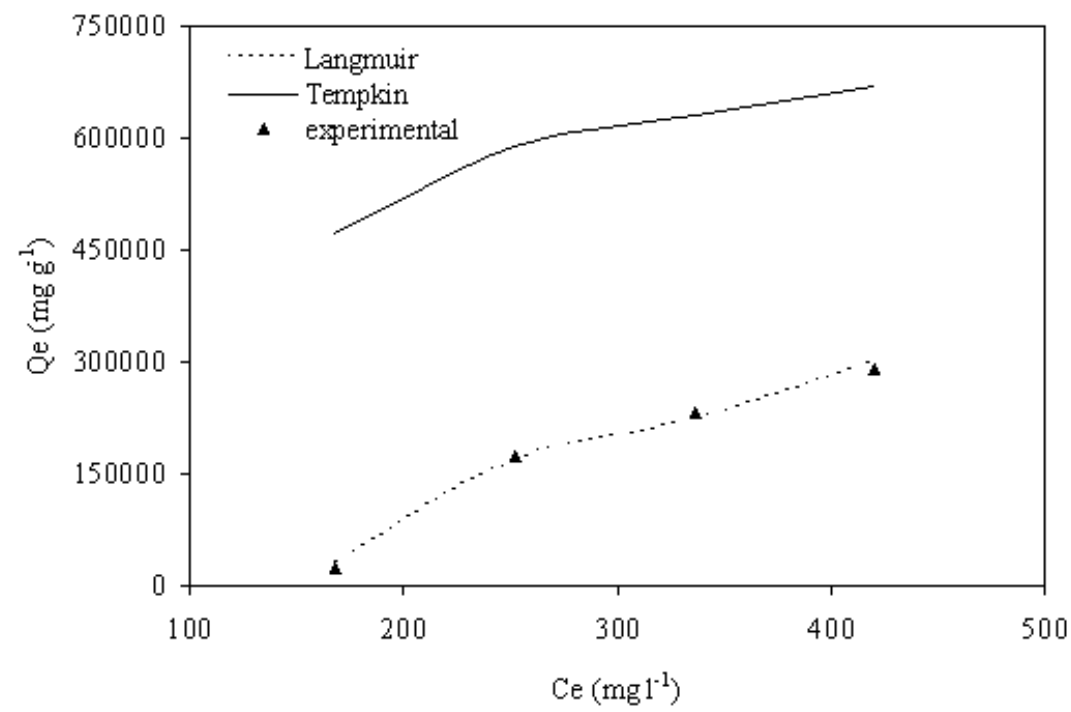

Figure 5. Comparison of isotherm model predictions with the experimental observation. 